\title{
The contribution of INTEGRAL to blazar science
}

\section{Luigi Foschini ${ }^{* \dagger}$ and Valentina Bianchin}

INAF/IASF-Bologna, Via Gobetti 101, 40129, Bologna (Italy)

E-mail: foschini@iasfbo.inaf.it

We review 6 years of INTEGRAL observations of blazars, from Target-Of-Opportunity (TOO) to normal observations to coordinated campaigns, from the new and unexpected discoveries to the improvements in this research field. Twenty blazars were detected to date, mainly (13) flatspectrum radio quasars (FSRQ), 6 of them with $z>2$. The major advantage of INTEGRAL is the large field of view of its instruments. This made possible to perform some interesting serendipitous discoveries.

7th INTEGRAL Workshop

September 8-11 2008

Copenhagen, Denmark

*Speaker.

${ }^{\dagger}$ A great thank you for comments, notes and suggestions to (in alphabetical order): V. Beckmann, E. Bottacini, M. Chernyakova, M. Chiaberge, W. Collmar, L. Costamante, T.J.-L. Courvoisier, M. Giroletti, M. Gliozzi, G. Lichti, L. Ostorero, E. Pian, R.M. Sambruna, M. Türler, M. Villata. 


\section{Introduction}

It is generally accepted that blazars are active galactic nuclei with a relativistic jet pointed toward the Earth with small angles $\left(<10^{\circ}\right)$, so that the relativistic motion can account for negligible $\gamma$-ray attenuation [40]. Blazars are organized along a "sequence" depending on the characteristics of their spectral energy distribution (SED), where flat-spectrum radio quasars (FSRQ) lie on the most luminous side and BL Lac Objects define the low luminosity side [18, 19]. Several challenges were posed to the sequence (e.g. [30]) leading to a recent general revision and improvement $[20,27]$. Specifically, while the original sequence was based on observational inputs (bolometric luminosity, shape of the SED), the recent improvements found their pillars in the physical parameters, like the mass of the central spacetime singularity and its accretion rate [20].

However, despite of this general view, there are still several open questions to understand the nature of these sources, like:

- where and how $\gamma$-rays are generated;

- what is the dependence of the radiation emission on the viewing angle (and therefore what is the link with radiogalaxies, if any);

- what is the jet composition;

- how the jet is coupled with the accretion disk;

- what are the scaling laws (link with microquasars);

just to mention a few. Observations in the X-rays and hard X-rays are crucial to investigate these issues, since they probe the part of the SED where synchrotron and inverse-Compton emissions are competing (see Fig. 1).

Here we review the main contributions of INTEGRAL [44] to the blazar science, obtained during the observing campaigns performed from the launch on October 17, 2002, to date.

\section{The INTEGRAL catalogs of point sources}

Two point source catalogs built with INTEGRAL data are currently available [25, 4]. The first one contains 400 sources and it is a true all sky survey, since it includes all the data from December 2002 (rev 25) to June 2006 (rev 463) plus a series of 13 exposures explicitly required during AO periods to complete the all-sky survey (200 ks, PI Churazov) [25]. The second catalog contains 421 sources detected in the period from November 2002 (rev 12) to April 2006 (rev 429) [4]. We integrated these lists by searching in the available literature, since several blazars have been recently observed with specific campaigns and therefore are not yet in these catalogs. In addition, there are also a few cases of blazars not present in the catalogs and, probably, the reason is that since they are generally built by performing source detection on very long exposure maps, this resulted in missing the short-term variability related to strong flares (if the blazar has too low flux when in quiescence and exceeds the instrument detection threshold only during short timescales).

A summary of all the blazars detected to date with INTEGRAL is reported in Table 1. 


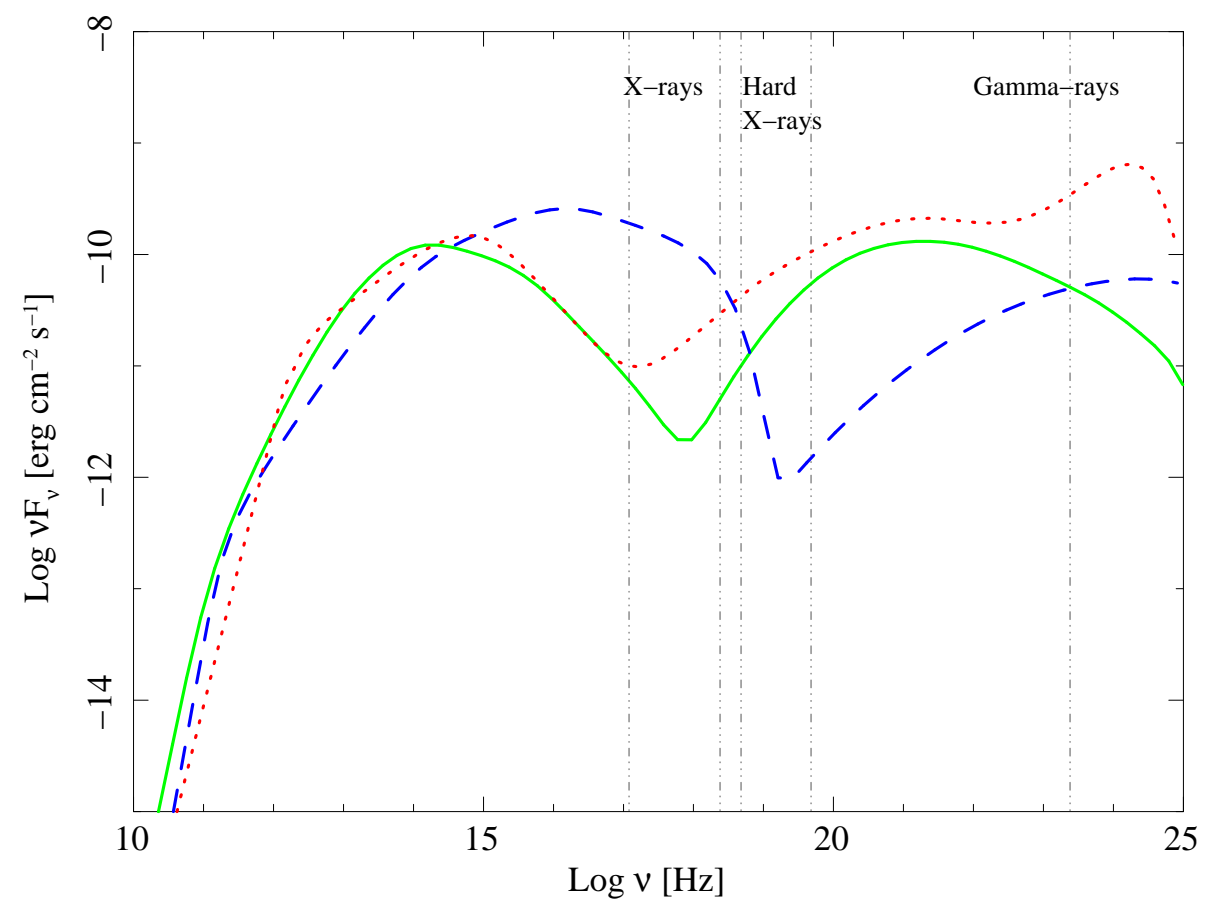

Figure 1: Typical SED of blazars: the red dotted line is the typical SED of Flat-Spectrum Radio Quasars (FSRQ); the green continuous line is for Low-Frequency Peaked BL Lac Objects (LBL); the dashed blue line is for High-Frequency Peaked BL Lac Objects (HBL). Grey vertical lines separate the X-rays ( $E \approx 0.5-10 \mathrm{keV}$ : e.g. XMM-Newton, Swift, Chandra, Suzaku), hard X-rays $(E \approx 20-200 \mathrm{keV}$ : e.g. INTEGRAL, Swift, Suzaku) and $\gamma$-rays domains (MeV-GeV-TeV ranges: Fermi, AGILE, MAGIC, HESS, VERITAS, CANGAROO). Example models are courtesy of G. Ghisellini.

\section{Some specific notes}

Here we shortly review the studies of specific or well-known sources based on INTEGRAL data. These notes do not match exactly the list in Table 1, since not all the blazars detected by INTEGRAL resulted in one or more dedicated papers. The source list is reviewed in order of ascending right ascension.

\subsection{PKS 0537-286 $(\mathrm{z}=3.1)$}

This is the second highest redshift FSRQ detected by INTEGRAL (the farthest is MG3 J225155+ 2217, see Sect. 3.15). Preliminary results presented at the present workshop showed a weak detection $(5 \sigma)$ in the hard X-ray band and a spectral flattening at soft X-ray energies [6].

\section{$3.2 \mathrm{S5} 0716+714(\mathrm{z}=0.31)$}

This LBL was the target of two multiwavelength campaigns. The first one, performed in November 2003, was organized by the ENIGMA-WEBT network (PI S. Wagner), when the source was extremely bright at radio frequencies, but optically faint $(R=14.17-13.64)$. No detection at hard X-rays was found [29], but it is worth noting that just a few days before this campaign, INTEGRAL was knocked down by a strong solar flare, which caused the instruments to switch off. 


\begin{tabular}{llccl}
\hline Blazar & Type & $\begin{array}{c}\text { Krivonos' Catalog } \\
{[25]}\end{array}$ & $\begin{array}{c}\text { Bird's Catalog } \\
{[4]}\end{array}$ & $\begin{array}{l}\text { Other } \\
\text { References }\end{array}$ \\
\hline RGB J0035+598 & HBL & Yes & Yes & \\
PKS 0537-286 & FSRQ & No & No & {$[6]$} \\
S5 0716+714 & LBL & No & No & {$[16,29,31]$} \\
S5 0836+71 & FSRQ & Yes & Yes & {$[31]$} \\
Mkn 421 & HBL & No & No & {$[26]$} \\
4C 04.42 & FSRQ & Yes & Yes & {$[15]$} \\
3C 273 & FSRQ & Yes & Yes & {$[2,10,13,39,36]$} \\
PKS 1241-399 & FSRQ & Yes & No & \\
3C 279 & FSRQ & Yes & Yes & {$[7,11,12]$} \\
1ES 1426+428 & HBL & No & No & {$[45]$} \\
Swift J1656.3-3302 & FSRQ & No & No & {$[28]$} \\
Mkn 501 & HBL & Yes & No & \\
NRAO 530 & FSRQ & No & No & {$[17]$} \\
PKS 1830-211 & FSRQ & Yes & Yes & {$[14,46]$} \\
PKS 1921-293 & FSRQ & No & Yes & \\
1ES 1959+650 & HBL & No & No & {$[5]$} \\
PKS 2149-307 & FSRQ & No & No & {$[3]$} \\
BL Lac & LBL & Yes & Yes & {$[32,42]$} \\
3C 454.3 & FSRQ & Yes & No & {$[1,27]$} \\
MG3 J225155+2217 & FSRQ & Yes & No & \\
\hline
\end{tabular}

Table 1: Summary of blazars detected with INTEGRAL during its 6 years of activity.

The second campaign was instead performed in April 2004, when high optical flux close to the historical maximum $(R=12.1)$ recorded at the end of March 2004, triggered simultaneous observations with INTEGRAL (PI Pian) and XMM-Newton (PI Tagliaferri). In this case, a faint detection $(4.5 \sigma)$ was measured in the $30-60 \mathrm{keV}$ energy band [31], consistent with the extrapolation from XMM-Newton data [16]. The studies on this outburst led to the conclusions that S5 $0716+714$ displayed two types of variability on long and short time scales [16]. On long time scales, there is only a change in flux without spectral variations. The gradual decay after the outburst can be due to the escape of electrons from the processing region or to a decrease of the seed photons. Modeling of the SED built with data obtained in 2004 and comparison with previous observations in quiescence (1996, see [22]) revealed that the physical parameters had negligible changes, except for the injected power. On the other hand, the short time scale variability is characterized by flux and spectral changes and it is likely to be due to variations in the slope of the electron distribution.

Interestingly, VLBI observations at $15 \mathrm{GHz}$ showed that, during the November 2003 campaign (no hard X-rays detection), the blazar core had the highest flux, while, in April 2004 (second campaign; detection with INTEGRAL), there was a peak in the emission from the knot A [8].

\section{$3.3 \mathrm{S5} 0836+710(\mathrm{z}=2.172)$}

The observation of this high-redshift flat-spectrum radio quasar was a "gift" from the large 
field of view (FOV $=29^{\circ} \times 29^{\circ}$ at zero response) of the IBIS imager onboard INTEGRAL. Indeed, S5 $0836+710$ is $6.5^{\circ}$ far from S5 $0716+714$, which was the target of the two above mentioned campaigns, and therefore it was detected as well. Comparison with previous observations with BeppoSAX [37], indicate that the flux was about a factor 3 lower, but the photon index remained constant within the measurement errors [31].

\subsection{Mkn $421(\mathrm{z}=0.03)$}

The INTEGRAL campaign of June 2006 (PI Lichti) on this famous HBL was triggered by an exceptional X-ray flux (up to $80 \mathrm{mCrab}$ measured by the All-Sky Monitor of RXTE in the $2-10 \mathrm{keV}$ energy band). The source was significantly detected with all the instruments onboard INTEGRAL (except SPI, which was annealing) and the SED, built with multiwavelength data, clearly showed the shift of the synchrotron peak from soft to hard X-rays [26]. The study of the variability across about 10 days of exposure revealed several flares, but only one showed significant spectral changes [26].

\section{$3.54 \mathrm{C} 04.42(\mathrm{z}=0.965)$}

The XMM-Newton and INTEGRAL observation of this FSRQ revealed a hard spectrum $(\Gamma \sim$ 1.2) extending to high-energy and a soft excess at low energies $(E<2 \mathrm{keV})$, which was interpreted as evidence of bulk motion Comptonization [15].

\section{6 $3 \mathrm{C} 273(\mathrm{z}=\mathbf{0 . 1 5 8})$}

3C 273 is perhaps the most ever known blazar, since the historical observations by Marteen Schmidt in 1963 [35] (see also [36] for a recent review). It was the first blazar observed by INTEGRAL, after the Performance Verification Phase on January 5, 2003 (rev 28, PI Courvoisier) [13]. This source was periodically observed during the INTEGRAL lifetime, often in coordination with $X M M-N e w t o n$ and RXTE [10]. The available data over the period $2003-2005$ suggest a possible anticorrelation between hard $(20-40 \mathrm{keV})$ and soft $(0.2-1 \mathrm{keV}) \mathrm{X}$-rays [2]. In 2004, during a historical minimum at submm wavelengths, a broad iron emission line was detected at $6.4 \mathrm{keV}$ [39].

\section{$3.73 C 279(\mathrm{z}=0.536)$}

This is another well known blazar and was the target of two multiwavelength campaigns: one in 2003 and the other in 2006 (PI Collmar in both cases) [11, 12]. While the first campaign was planned independently from the status of the source, the second one was triggered by high optical flux observed by ground telescope at the beginning of January 2006 (see also [7]). By comparing the SED obtained from the two campaigns, the most striking feature is that, despite a strong change in optical fluxes (about one order of magnitude), little or negligible changes occurred in hard X-rays $[12,7]$. The theoretical implications are still to be understood.

\subsection{ES 1426+428 (z=0.129)}

This is an extreme HBL, with the synchrotron emission probably peaking in the hard X-rays. INTEGRAL observation performed in May 2006 (PI Wolter) showed a rather flat photon index for a HBL $(\Gamma \sim 2)$, confirming its unusual characteristics [45]. 


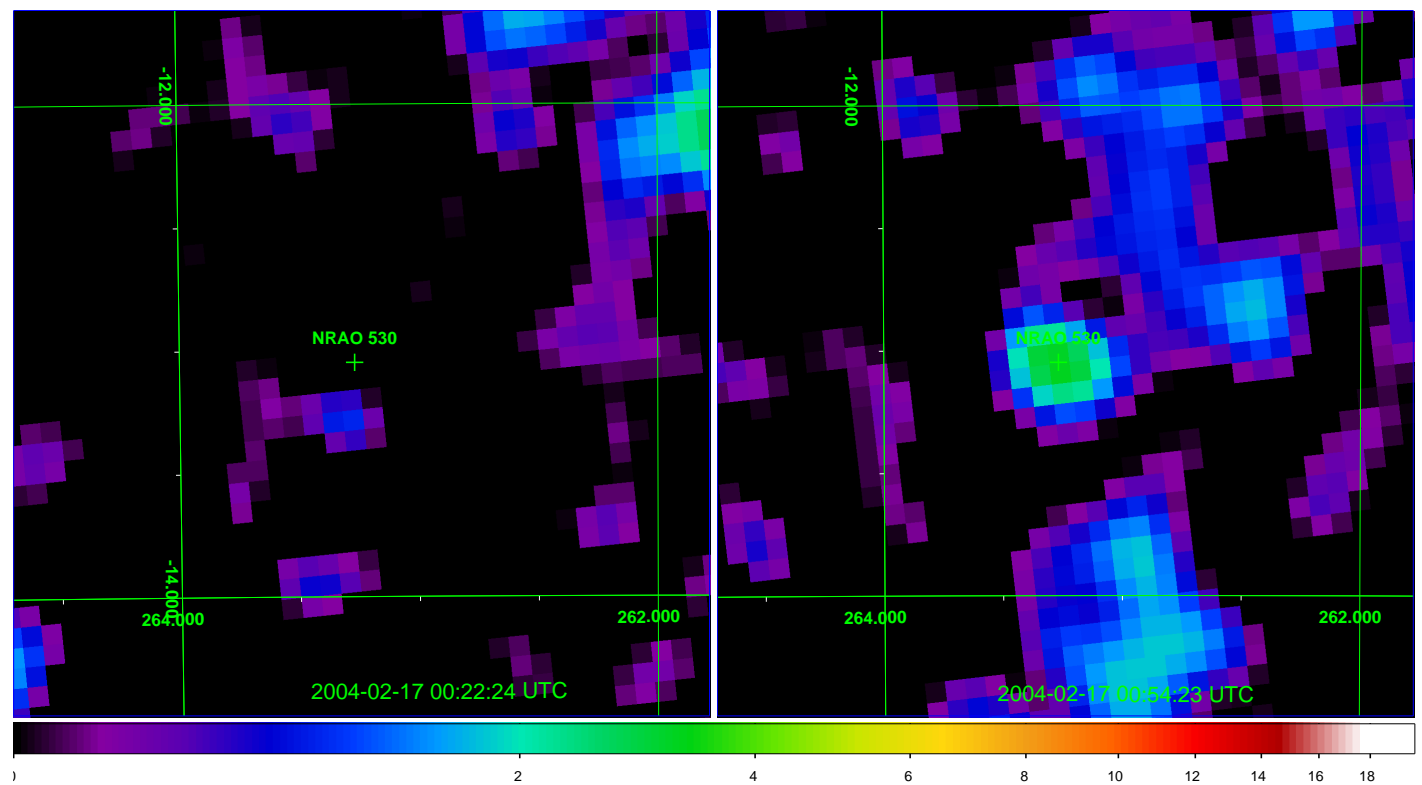

Figure 2: The short and strong hard X-ray flare observed from NRAO 530 on February 17, 2004. This is a sequence of two consecutive pointings $(\approx 2 \mathrm{ks}$ each $)$ of IBIS/ISGRI in the $20-40 \mathrm{keV}$ energy band, showing that the source suddenly appeared and disappeared (zoom of the image in [17]).

\subsection{Swift J1656.3-3302 ( $\mathrm{z}=2.40)$}

This high-redshift blazar was discovered by Swift and later confirmed by INTEGRAL. The high-energy part of the spectrum displayed a photon index of about 1.6, but with a spectral flattening at low energies $(E<2 \mathrm{keV})$, which was interpreted as absorption intrinsic to the source [28].

\subsection{NRAO $530(\mathrm{z}=0.902)$}

The importance of having a large FOV is again underlined in this case: NRAO 530 had a strong and short flare that was serendipitously detected by INTEGRAL during the normal operations of the Galactic Centre Deep Exposure on February 17, 2004. The $20-40 \mathrm{keV}$ flux emitted by the blazar increased up to $\approx 2 \times 10^{-10} \mathrm{erg} \mathrm{cm}^{-2} \mathrm{~s}^{-1}$ and then dropped below the instrument detection limit in less than 1 hour (see Fig. 2) [17]. Given such extraordinary behaviour, never observed in a flatspectrum radio quasar, there was the doubt of a contaminating source within the $3^{\prime}$ error circle of IBIS. However, a couple of snapshots with the X-Ray Telescope (XRT) onboard Swift revealed that NRAO 530 is the only high-energy source present in the IBIS error circle. In addition, this blazar is known to display high-amplitude variability at almost all the wavelengths and therefore, the flux measured by INTEGRAL is not at odds with historical observations.

\subsection{PKS 1830-211 $(\mathrm{z}=2.507)$}

This is a gravitationally lensed FSRQ and therefore the jet radiation, in addition to the Doppler boosting, is amplified also by the gravitation of the intervening galaxy at $z=0.89$. The analysis of the SED built with Chandra, INTEGRAL and EGRET data (not simultaneous) favours the typical 


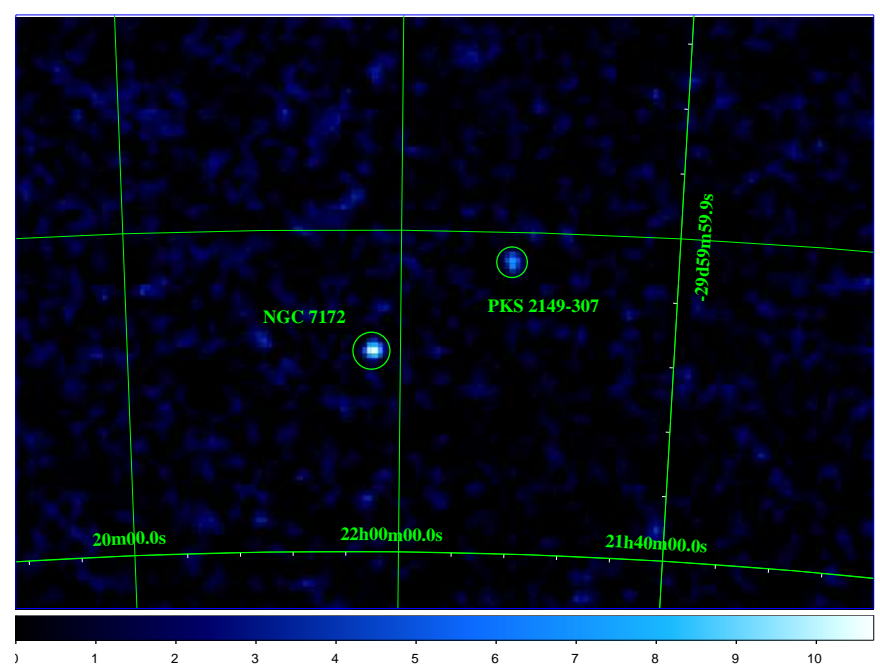

Figure 3: IBIS/ISGRI significance map in the $20-40 \mathrm{keV}$ energy band of the region around NGC 7172 with the serendipitous detection of PKS $2149-307$.

emission mechanism powering FSRQ, with a spectral flattening at soft X-rays $(E<4 \mathrm{keV})$ that was initially interpreted as ionized absorption intrinsic to the source [14]. However, a more recent study on a SED built with XMM-Newton, INTEGRAL, COMPTEL and EGRET data (not simultaneous) with the highest statistics available to date, strongly supported the hypothesis that the soft X-ray spectral flattening is the natural curvature of the FSRQ continuum [46].

\subsection{ES 1959+650 $(\mathrm{z}=0.047)$}

This HBL was detected during the observations of the Key Programme on the North Ecliptic Pole region, with a flux about one half of that displayed in the 2001 outburst, but with a hard photon index of $\approx 1.9[5]$.

\subsection{PKS 2149-307 ( $\mathrm{z}=2.345)$}

This high-redshift blazar was detected serendipitously in 2004 during an observation of the nearby Seyfert 2 active nucleus NGC 7172 (distance $\approx 2.6^{\circ}$, see Fig. 3). The INTEGRAL/IBIS spectrum in the $20-100 \mathrm{keV}$ has the same shape of that detected in 2005 by Swift/BAT during its first nine month of operations [34], but it is a factor two lower [3]. Therefore, over about one year, the hard X-ray flux of this blazar roughly doubled its value, without changes in slope.

\subsection{C $454.3(\mathrm{z}=0.859)$}

This FSRQ has been extremely active since 2005 and several multiwavelength campaigns were performed. In May 2005, 3C 454.3 underwent a long and intense outburst observed by many ground based telescopes (WEBT [43]) and satellites (Swift [23], Chandra [43]). Also INTEGRAL was activated and pointed to the blazar on May $15-18,2005$ (PI Pian) [32]. This observation can be better understood in the framework of the "jet economic model" [24] and by comparing the source evolution over several years [21]. According to this model, the ratio between Synchrotron Self-Compton (SSC) and External Compton (EC) emission is regulated by the bulk Lorentz factor 


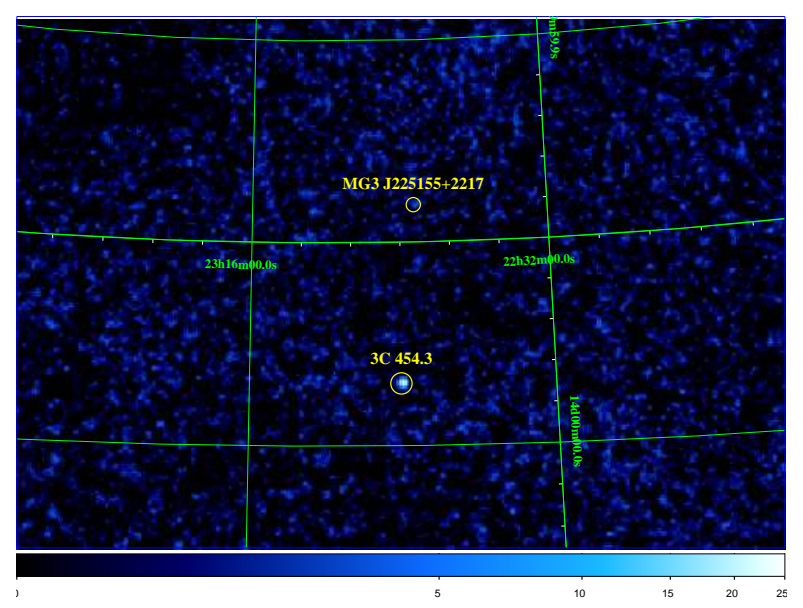

Figure 4: IBIS/ISGRI significance map in the $20-40 \mathrm{keV}$ energy band of the region around $3 \mathrm{C} 454.3$ and with the blazar MG3 J225155 + 2217 .

$\Gamma$, which in turn is linked to the compactness of the source. This means that changes in the shape of the SED can be explained with an almost constant jet power and with different places where the main dissipation occurs. In 2005, the high hard X-rays flux observed by INTEGRAL can be modeled with low $\Gamma$ and high magnetic field, favouring the hypothesis that the main dissipation occurred in a compact region, where the SSC dominates and the EC gave little contribution. Instead, in 2007, a high $\gamma$-ray flux observed by AGILE $([9,41])$ together with moderately low optical and $\mathrm{X}$-ray fluxes, suggest that the dissipation occurred at larger distances, therefore with high $\Gamma$ and low magnetic field [21].

3C 454.3 continued to be active in 2007 (and it is still very active also in 2008, displaying an exceptional outburst observed by Fermi/LAT [38]), so that another multiwavelength campaign involving INTEGRAL was triggered by the AGILE team in November 2007 (PI Vercellone). The $20-200 \mathrm{keV}$ flux was about one order of magnitude less than that detected in 2005, while the $\gamma$-ray flux was quite high [42], favouring again the "jet economic model" interpretation.

\subsection{MG3 J225155+2217 (z=3.668)}

Yet another gift from the large FOV of INTEGRAL. This source was first serendipitously detected in the FOV of 3C 454.3 observation in May 2005 (distance $\approx 6^{\circ}$; see Fig. 4) and reported as unidentified source in the Krivonos' Catalog [25]. Later, it was associated with the high-redshift $(z=3.668)$ blazar MG3 J225155+2217 [1] and it is the farthest detected by INTEGRAL to date. The modeling of the SED showed that the emission from this source is largely dominated by the inverse-Comtpon component [27], but still in the framework of a typical high-redshift FSRQ (like, e.g., Swift J0746.3 +2548 at $z=2.979$ [33]).

\section{Final remarks}

During about 6 years of activity, INTEGRAL detected 20 blazars, with a strong preference to FSRQ (13 sources) and, particularly, 6 FSRQ have redshift greater than 2. The major strength of INTEGRAL is the large FOV of its instruments (specifically IBIS, with more than 800 square 
degrees), which allowed us to perform some important serendipitous discoveries, like the highredshift extreme blazar MG3 J225155 + 2217 and the short and strong flare of NRAO 530. The short term variability can be studied only in a few cases, for the brightest sources, but the sensitivity that can be reached with long/deep exposures is sufficient to probe the moderately high-redshift Universe. These assets can be emphasized by the Key Programmes, which are now (from AO7) constituting $70 \%$ of the observing time.

INTEGRAL gave interesting contributions also in studying blazars in outburst, although its contribution is "diluted" in the multiwavelength campaigns. In addition, given the erratic and unpredictable occurrence and duration of these outbursts, it is quite difficult to catch the right time, with the maximum flux, in order to have data with the highest statistics. Nevertheless, great synergies are expected with Fermi, AGILE, MAGIC II and the forthcoming HESS II.

For the studies of hard X-ray emission from blazars in the next decade, it is expected that the legacy of INTEGRAL will be received by Simbol-X, NuStar, Astro- $H$ and, hopefully, by the International X-ray Observatory (IXO).

\section{References}

[1] Bassani L. et al., IGR J22517+2218 = MG3 J225155+2217: A New Gamma-Ray Lighthouse in the Distant Universe, ApJ 669 (2007) L1.

[2] Bianchin V. et al., Three years monitoring of 3C 273 with INTEGRAL and XMM-Newton, Mem. SAIt, 79 (2008) 232.

[3] Bianchin V. et al., The changing look of PKS 2149-306, A\&A, (2009), accepted for publication.

[4] Bird A.J. et al., The Third IBIS/ISGRI Soft Gamma-Ray Survey Catalog, ApJS 170 (2007) 175.

[5] Bottacini E. et al., Blazar 1ES 1959+650 shows high and hard X-ray state, ATel 1315 (2007).

[6] Bottacini E. et al., PKS 0537-286, the high redshift FSRQ, giving new insights into the jet physics, Proceedings of the 7th INTEGRAL Workshop: An INTEGRAL View of Compact Objects, IPoS (Integraio8) 066 ' (2009).

[7] Böttcher M. et al., The WEBT Campaign on the Blazar 3C 279 in 2006, ApJ 670 (2007) 968.

[8] Britzen S. et al., “Oscillating” components in the BL Lac object 0716+714?, in Proceedings of the 8th European VLBI Network Symposium, (2006),

[9] Bulgarelli A. et al., AGILE pointing at 3C 454.3: end of the observations and preliminary results, ATel 1167, (2007).

[10] Chernyakova M. et al., 2003-2005 INTEGRAL and XMM-Newton observations of 3C 273, A\&A 465 (2007) 147.

[11] Collmar W. et al., Multifrequency Observations of the Gamma-Ray Blazar 3C 279 in Low-State During INTEGRAL AO-1, in Proceedings of the $5^{\text {th }}$ INTEGRAL Workshop: The INTEGRAL Universe (ESA SP-552). Eds V. Schönfelder, G. Lichti \& C. Winkler, (2004) p. 555.

[12] Collmar W. et al., Multifrequency Observations of the Blazar 3 C 279 in January 2006, in Proceedings of the $6^{\text {th }}$ INTEGRAL Workshop: The Obscured Universe (ESA SP-622). Eds S. Grebenev, R. Sunyaev \& C. Winkler, (2007) p. 207.

[13] Courvoisier T.J.-L. et al., Simultaneous observations of the quasar 3C 273 with INTEGRAL and RXTE, A\&A 411 (2003) L343. 
[14] De Rosa A. et al., The broad-band X-ray spectrum of the blazar PKS B1830-211 by Chandra and INTEGRAL, A\&A 438 (2005) 121.

[15] De Rosa A. et al., Bulk Compton motion in the luminous quasar 4C04.42?, MNRAS 388 (2008) L54.

[16] Foschini L. et al., Simultaneous X-ray and optical observations of S5 0716+714 after the outburst of March 2004, A\&A 455 (2006) 871.

[17] Foschini L. et al., A short hard X-ray flare from the blazar NRAO 530 observed by INTEGRAL, A\&A 450 (2006) 77.

[18] Fossati G. et al., A unifying view of the spectral energy distributions of blazars, MNRAS 299 (1998) 433.

[19] Ghisellini G. et al., A theoretical unifying scheme for gamma-ray bright blazars, MNRAS 301 (1998) 451.

[20] Ghisellini G. \& Tavecchio F., The blazar sequence: a new perspective, MNRAS 387 (2008) 1669.

[21] Ghisellini G. et al., On the 2007 July flare of the blazar 3C 454.3, MNRAS 382 (2007) L82.

[22] Giommi P. et al., Synchrotron and inverse Compton variability in the BL Lacertae object S5 0716+714, A\&A 351 (1999) 59.

[23] Giommi P. et al., Swift and infra-red observations of the blazar 3C 454.3 during the giant X-ray flare of May 2005, A\&A 456 (2006) 911.

[24] Katarzyński K. \& Ghisellini G., Dissipation of jet bulk kinetic energy in powerful blazars, A\&A 463 (2007) 529.

[25] Krivonos R. et al., INTEGRAL/IBIS all-sky survey in hard X-rays, A\&A 475 (2007) 775.

[26] Lichti G. et al., INTEGRAL observations of the blazar Mrk 421 in outburst. Results of a multi-wavelength campaign, A\&A 486 (2008) 721.

[27] Maraschi L. et al., Testing the blazar spectral sequence: X-ray selected blazars, MNRAS, 391, (2008), 1981.

[28] Masetti N. et al., High-redshift blazar identification for Swift J1656.3-3302, A\&A 480 (2008) 715.

[29] Ostorero L. et al., Testing the inverse-Compton catastrophe scenario in the intra-day variable blazar S5 0716+71. I. Simultaneous broadband observations during November 2003, A\&A 451 (2006) 797.

[30] Padovani P., The blazar sequence: validity and predictions, Astrophys. Space Sci. 309 (2007) 63.

[31] Pian E. et al., INTEGRAL observations of the field of the BL Lacertae object S5 0716+714, A\&A 429 (2005) 427.

[32] Pian E. et al., INTEGRAL observations of the blazar 3C 454.3 in outburst, A\&A 449 (2006) L21.

[33] Sambruna R.M. et al., Discovery of an Extreme MeV Blazar with the Swift Burst Alert Telescope, ApJ 646 (2006) 23.

[34] Sambruna R.M. et al., Swift Observations of High-Redshift Radio-loud Quasars, ApJ 669 (2007) 884.

[35] Schmidt M., 3C 273 : A Star-Like Object with Large Red-Shift, Nature 197 (1963) 1040.

[36] Soldi S. et al., The multiwavelength variability of 3C 273, A\&A 486 (2008) 411.

[37] Tavecchio F. et al., Gamma-loud Quasars: A View with BeppoSAX, ApJ 543 (2000) 535. 
[38] Tosti G. et al., GLAST-LAT detection of extraordinary gamma-ray activity in 3C 454.3, ATel 1628, (2008).

[39] Türler M. et al., A historic jet-emission minimum reveals hidden spectral features in 3C 273, A\&A 451 (2006) L1.

[40] Urry C.M. \& Padovani P., Unified schemes for radio-loud active galactic nuclei, PASP 107 (1995) 803.

[41] Vercellone S. et al., AGILE gamma-ray detection of the Blazar 3C 454.3, ATel 1160, (2007).

[42] Vercellone S. et al., Multiwavelength observations of 3C 454.3. I. The AGILE 2007 November campaign on the "Crazy Diamond”, ApJ, 690, (2009), 1018.

[43] Villata M. et al., The unprecedented optical outburst of the quasar 3C 454.3. The WEBT campaign of 2004-2005, A\&A 453 (2006) 817.

[44] Winkler C. et al., The INTEGRAL mission, A\&A 411 (2003) L1.

[45] Wolter A. et al., The Hard Synchrotron X-ray Spectrum of the TeV BL Lac IES 1426+428, in Proceedings of Extragalactic Jets: Theory and Observation from Radio to Gamma Ray, ASP Conference Series, Vol. 386, Eds Travis A. Rector \& David S. De Young, (2008), p. 302.

[46] Zhang S. et al., High-Energy Properties of PKS 1830-211, ApJ 683 (2008) 400. 